\title{
Mechanics of a process to assemble microspheres on a patterned electrode
}

\section{Citation}

Zhu, Ting, Zhigang Suo, Adam Winkleman, and George M. Whitesides. 2006. “Mechanics of a Process to Assemble Microspheres on a Patterned Electrode." Applied Physics Letters 88 (14): 144101. https://doi.org/10.1063/1.2191743.

\section{Permanent link}

http://nrs.harvard.edu/urn-3:HUL.InstRepos:41467477

\section{Terms of Use}

This article was downloaded from Harvard University's DASH repository, and is made available under the terms and conditions applicable to Other Posted Material, as set forth at http:// nrs.harvard.edu/urn-3:HUL.InstRepos:dash.current.terms-of-use\#LAA

\section{Share Your Story}

The Harvard community has made this article openly available.

Please share how this access benefits you. Submit a story.

Accessibility 


\title{
Mechanics of a process to assemble microspheres on a patterned electrode
}

\author{
Ting Zhu ${ }^{\text {a) }}$ and Zhigang Suo ${ }^{\text {b) }}$ \\ Division of Engineering and Applied Sciences, Harvard University, Cambridge, Massachusetts 02138 \\ Adam Winkleman and George M. Whitesides ${ }^{c}$ \\ Department of Chemistry and Chemical Biology, Harvard University, Cambridge, Massachusetts 02138
}

(Received 11 December 2005; accepted 15 February 2006; published online 3 April 2006)

\begin{abstract}
A process has been demonstrated recently to assemble microspheres on a patterned electrode under the influence of an applied voltage. Here we examine the mechanics of this process, and describe both the conditions under which excess microspheres jump off the electrode when the voltage is applied, and the forces that attract the remaining microspheres to the desired positions. A quantitative mechanistic understanding of this process rationalizes experimental observations, provides scaling relations, and suggests modifications of the process. (c) 2006 American Institute of
\end{abstract} Physics. [DOI: 10.1063/1.2191743]

An objective of growing importance in integrated circuit technology is to develop processes that produce low-cost, large-area electronic systems. ${ }^{1-4}$ Understanding how to bridge different sizes in fabrication-from $50 \mathrm{~nm}$ scale transistors to meter-scale displays-remains a challenge. One approach is to fabricate small components in one set of processes and to assemble them in a second. ${ }^{4-6}$ Conventional pick-and-place technology-whether by humans or robots - is costly and slow when the system consists of a large number of components, and especially small components $(1-100 \mu \mathrm{m})$. Massively parallel processes are needed to produce electronic systems that would otherwise be impossible.

Figure 1 illustrates a process we have described that uses electrostatic interactions to assemble small components into a desired pattern. ${ }^{6}$ The parameters indicated in the figure caption were determined experimentally by trial and error. This Letter describes a mechanistic understanding of the process.

The gold film, the polystyrene (PS) plate, and the metal support beneath the plate form a parallel-plate capacitor. When subject to an applied voltage $U$, a uniform electric field builds up inside the PS plate. A fringe field also develops outside the PS plate (Fig. 2), where $D$ is the diameter of the biased electrode and $t$ is the thickness of the PS plate. We carried out the calculation with an axis-symmetric model, using the finite element program FEMLAB. ${ }^{7}$ Just above the gold film, the electric field on the surface decreases from the edge towards the center, where the electric field reaches a minimum and scales as $E_{a} \sim U / D .{ }^{8}$ Below the gold film, the electric field is given by $E_{b}=U / t$. Because $D \gg t$, we conclude that $E_{a} \ll E_{b}$.

The electric field above the biased electrode was sufficiently high that the glass spheres were charged, as confirmed by measurements of their charges with a Faraday cup. We assume that the glass spheres are initially charged to the same electric potential as the gold film, and will assess the

\footnotetext{
a) Present address: Woodruff School of Mechanical Engineering, Georgia Institute of Technology, Atlanta, GA 30332.

b)Electronic mail: suo@deas.harvard.edu

${ }^{c)}$ Electronic mail: gwhitesides@ gmwgroup.harvard.edu
}

correctness of this assumption later by comparing its predictions with experimental observations. In a medium of permittivity $\varepsilon$, near the surface of a particle of constant potential, the electric field $E$ is normal to the surface and points either toward or away from the particle. This electric field exerts on the surface of the particle the Maxwell stress $\sigma=\varepsilon E^{2} / 2$; this stress is normal to the surface of the particle and points away from the particle. ${ }^{9}$ The integration of the Maxwell stress over the surface gives the net force on the particle.

We first consider the conditions under which the excess glass spheres jump away from the electrode. Each excess sphere is shielded from the electric field $E_{b}$ by the gold film and the spheres over the windows, but gains an upward force from the electric field $E_{a}$. The minimum upward force occurs at the center of the gold film and scales as $F_{E} \sim \varepsilon_{0} E_{a}^{2} d^{2}$ $\sim \varepsilon_{0}(U / D)^{2} d^{2}$, where $\varepsilon_{0}$ is the permittivity of vacuum. The weight of each sphere is $F_{g}=\pi \rho g d^{3} / 6$, where $\rho$ is the density of the sphere and $g$ is the gravitational acceleration. The (a)

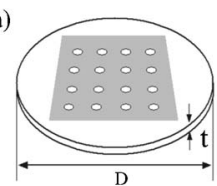

(c)

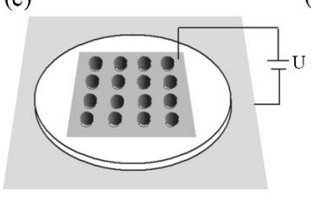

(b)

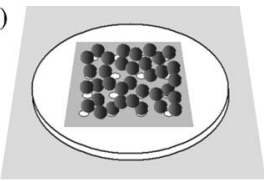

(d)
FIG. 1. (Color online) The process to assemble spheres on a patterned electrode. (a) On a plate of polystyrene (PS; diameter $D=20 \mathrm{~mm}$ and thickness $t=1 \mathrm{~mm}$ ), we patterned a $45 \mathrm{~nm}$ thick gold film with circular windows, which exposed the PS surface underneath. (b) We then placed the PS plate on a metal support and poured glass spheres of diameter $d \sim 100 \mu \mathrm{m}$ on top of the gold film. The diameter of each glass sphere was comparable to that of a window, but the spheres outnumbered the windows by a factor of at least 10. (c) When we applied a voltage $U \sim 10 \mathrm{kV}$ between the gold film and the metal support, the excess spheres jumped off the gold film and left every window covered with one sphere (with occasional defects, such as uncovered windows or excess spheres). The process positioned the spheres over the windows over the entire surface $\left(\sim 4 \mathrm{~cm}^{2}\right)$ in $\sim 5 \mathrm{~s}$, with $\sim 1 \%$ defect. (d) An optical micrograph of an array of assembled microspheres. 


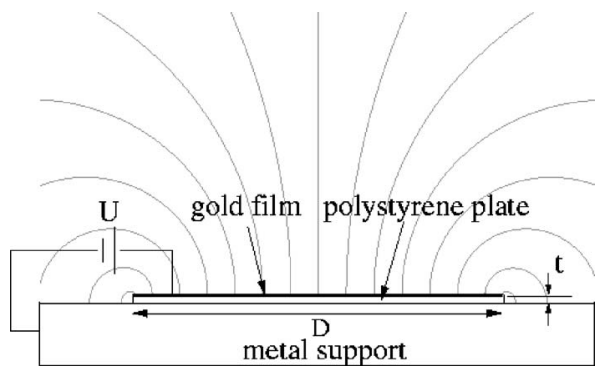

FIG. 2. (Color online) Electric field lines showing the distribution of fringe field outside the PS plate. The calculation was carried out with voltage $U$ applied between the gold film and the metal support, where $D$ is the diameter and $t$ is the thickness of the polystyrene plate, $D / t=40$. The electric field is uniform and intense inside the polystyrene plate (not shown). To visualize the electrostatic field at the scale of the capacitor, we neglect the windows and spheres, which would only influence the field at a scale comparable to the diameter of the spheres.

sphere jumps away from the electrode when $F_{E}>F_{g}$; this inequality gives the scaling relation for the minimum voltage,

$$
U_{\min } \sim D \sqrt{d \rho g / \varepsilon_{0}} .
$$

Using representative values, $\rho=2.44 \times 10^{3} \mathrm{~kg} / \mathrm{m}^{3}$, $d=100 \mu \mathrm{m}$, and $D=20 \mathrm{~mm}$, Eq. (1) gives an order of magnitude estimate, $U_{\min } \sim 10 \mathrm{kV}$; this estimate is consistent with the experimental observation. Here we have ignored adhesion between the microspheres and gold film; both our study and previous studies ${ }^{10,11}$ have shown that adhesion only becomes important for spheres less than about $10 \mu \mathrm{m}$ in diameter. This conclusion, however, may be altered by high humidities or patterned surfaces; we have not studied the effect of adhesion in this process.

We next consider the electrostatic forces that attract the remaining spheres to the windows. In a parallel-plate capacitor, the attraction between the two electrodes causes a Maxwell stress $\varepsilon E_{b}^{2} / 2$, where $\varepsilon$ is the permittivity of PS. ${ }^{8}$ If the gold electrode were continuous and had no windows, the attractive force on a circular area of diameter $d$ of the gold electrode would be $F_{0}=\varepsilon(U / t)^{2} \pi d^{2} / 8$. In the experimental setup, however, the gold electrode contains windows, each of which is covered by an equipotential glass sphere. The electric field inside the capacitor exerts an attractive force $F$ on the sphere; this force $F$ scales with $F_{0}$, but should be smaller than $F_{0}$ because of the curvature of a sphere, it can only partially cover the window. For a given location of a sphere, we first solve the electrostatic field by using the finite element method and then calculate the force $F$ on the sphere by integrating the Maxwell stress. As an example of such a calculation, Fig. 3 shows that the attractive force is $F \approx 0.7 F_{0}$ when the sphere is adjacent to the window and decays rapidly when the sphere is further above the window. Additional calculations for particles of other shapes and at locations away from the center axis of the windows also showed that the electrostatic force is attractive and localized around the windows. For representative values, $U=10 \mathrm{kV}, t=1 \mathrm{~mm}$, $d=100 \mu \mathrm{m}$, and $\varepsilon=2.6 \varepsilon_{0}$, we find that $F_{0}=9.0 \mu \mathrm{N}$. By comparison, the weight of such a glass sphere is $F_{g}=0.013 \mu \mathrm{N}$. The electrostatic force attracts the sphere to the window; this force appears to be sufficient to overcome other forces such as friction that would prevent the sphere from moving laterally. A detailed study of this competition is beyond the scope of this Letter. Experimentally, we observed that the quality of Downloaded 31 May 2006 to 128.103.60.225. Redistribution subject

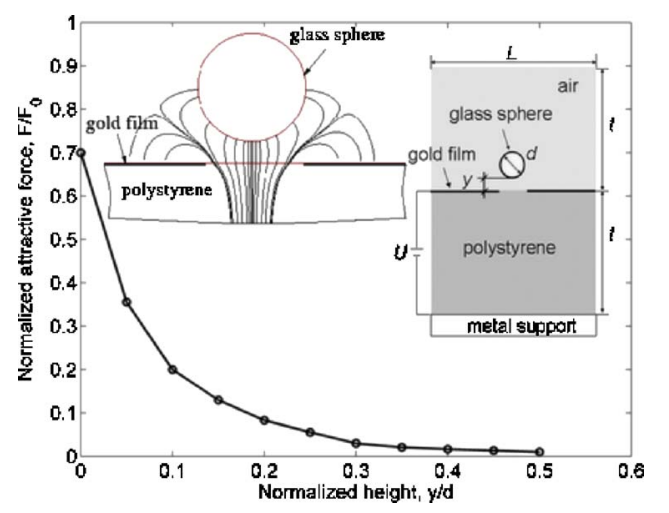

FIG. 3. (Color online) The attractive force on a glass sphere as a function of its height above a window. The calculation was carried out with an axisymmetric model $(L / d=4, t / d=10)$. The attractive force on the glass sphere is normalized by $F_{0}=\varepsilon(U / t)^{2} \pi d^{2} / 8$ (see the text). We prescribe the voltage $U$ between the gold film and the metal support, and a vanishing normal component of the electric displacement on each of the other surfaces. The insets show the distribution of electric field lines near the window (left) and the schematic of simulation cell (right), respectively.

assembly improved if we gently tapped the PS plate; this tapping presumably reduced the effects of friction and stiction.

The mechanistic picture rationalizes various experimental observations. For example, as the applied voltage increased and spheres were ejected from the pile on the surface, the pile shrunk from the periphery to the center of the gold film, indicating that the excess spheres at the periphery jumped away at a lower applied voltage than those at the center. This observation is consistent with the distribution of the electric field above the gold film: the electric field increases with the distance from the center of the gold film. In order to assemble spheres with a low rate of defects, our experiments also showed that the applied voltage must be within a certain range. If the applied voltage was too low, the fringe electric field above the gold film was insufficient to lift the spheres against gravitation, as discussed above. If the applied voltage was too high, many windows in the gold film remained vacant, and did not house spheres. This observation could also be interpreted within our model. Upon applying a large voltage, the surface of the PS windows might become charged; this charging could be from either charge injection ${ }^{12}$ or air ionization and deposition. ${ }^{13}$ Experimentally, we are able to visualize a corona at the corners of the gold electrode as well as observe the ionic breeze from the electrode when using the high voltages. These charges would shield the glass spheres on the windows from the electric field inside the capacitor, so that even these spheres could be lifted by the fringe electric field above the surface.

Our mechanistic picture also suggests improvements to the process. Equation (1) shows that the minimum voltage to lift spheres against gravity scales with the diameter $D$ of the biased electrode. Consequently, to assemble spheres over a large area, we would need a very high voltage. To shorten the path of the voltage drop, we placed a second metal plate at a height $H$ above the gold film, in addition to the metal support beneath the PS plate. We grounded the two metal plates and applied a voltage to the gold film. This arrangement of the electrodes was used to determine a minimum voltage for the excess spheres to jump off the gold film, but was not optimal for high-yield assemblies. With this geometry, the ejected spheres bounced off the upper electrode and returned to the to AIP license or copyright, see http://apl.aip.org/apl/copyright.jsp 
TABLE I. Experimental values of the minimum voltage to lift the excess spheres. A third electrode was placed distance $H$ above the gold film.

\begin{tabular}{cccc}
\hline \hline$H(\mathrm{~mm})$ & $U_{\min }^{\mathrm{glass}}(\mathrm{kV})$ & $U_{\min }^{\mathrm{Cu}}(\mathrm{kV})$ & $U_{\min }^{\mathrm{Cu}} / U_{\min }^{\mathrm{glass}}$ \\
\hline 5 & 1.1 & 1.4 & 1.27 \\
9 & 1.9 & 2.4 & 1.26 \\
11 & 2.4 & 2.9 & 1.21 \\
15 & 3.2 & 4.0 & 1.25 \\
20 & 5.2 & & \\
25 & 6.0 & & \\
\hline \hline
\end{tabular}

gold film only to repeat this process. ${ }^{11}$ This ricocheting of the ejected spheres disrupted the assembled arrays. The collisions against the upper grounded electrode need not to discharge the ejected glass spheres; they ricochet due to the mechanical collisions alone. Other geometries of the upper electrode (e.g., a nonparallel upper electrode ${ }^{10,14}$ or a mesh electrode ${ }^{15}$ ) would require a lower voltage and still yield low-defect assemblies.

Table I shows the measured minimum voltage $U_{\min }$ as a function of $H$. For glass spheres of diameter $75 \mu \mathrm{m}, U_{\min }$ can be reduced by about a factor of 5 as $H$ decreases from 25 to $5 \mathrm{~mm}$. Table I also includes measured values of $U_{\text {min }}$ for $\mathrm{Cu}$ spheres (diameter, $30 \mu \mathrm{m}$ ). We observe that $U_{\min }^{\mathrm{Cu}} / U_{\min }^{\text {glass }}$ is approximately a constant independent of the height of the second metal plate. The same constant ratio was also observed experimentally when we changed the diameter of the gold film.

We understand the constant ratio of $U_{\min }^{\mathrm{Cu}} / U_{\min }^{\text {glass }}$ as follows. For any geometry of electrodes, the arguments leading to Eq. (1) now give $U_{\min }=\lambda \sqrt{d \rho g / \varepsilon_{0}}$, where $\lambda$ is a length that measures the effective voltage path in a given design. This length $\lambda$ depends on the geometry of the electrodes, but is independent of the size and the material of the spheres, provided they are charged to the potential of the gold film. When spheres of different sizes and materials are assembled using the same configuration of the electrodes, the ratio of the minimum voltages required to lift the excess spheres is $U_{\min }^{\mathrm{Cu}} / U_{\min }^{\text {glass }}=\sqrt{\left(d^{\mathrm{Cu}} \rho^{\mathrm{Cu}}\right) /\left(d^{\text {glass }} \rho^{\text {glass }}\right)}$. Observe that this ratio is independent of the geometry of the electrodes. Taking $\rho^{\text {glass }}$ $=2.44 \times 10^{3} \mathrm{~kg} / \mathrm{m}^{3}, \quad d^{\text {glass }}=75 \mu \mathrm{m}, \rho^{\mathrm{Cu}}=8.92 \times 10^{3} \mathrm{~kg} / \mathrm{m}^{3}$, and $d^{\mathrm{Cu}}=30 \mu \mathrm{m}$, we obtain a theoretical value $U_{\min }^{\mathrm{Cu}} / U_{\min }^{\text {glass }}$ $=1.21$, which is in good agreement with the experimental values listed in Table I. This agreement between the minimum electric fields for different diameters of the biased electrode lends support to the two major assumptions in our model: the lifting condition is governed by the competition between the electrostatic and gravitational forces with negligible effect of adhesion, and the glass spheres are charged to the same electric potential as the gold electrode.

In summary, we have shown that the process developed by Winkleman et al. ${ }^{6}$ requires two electric fields: the field above the gold film $E_{a}$ to lift excess spheres against gravity and the field below the gold film $E_{b}$ to attract the spheres to the windows. Our scaling relation for the minimum voltage is consistent with the experimental data of both original and modified geometries of electrodes. This mechanistic understanding enables us to modify the process in many other ways to fulfill various design requirements and reinforces the notion that the electrostatic forces can rapidly assemble small objects over large areas.

This research was supported by the Army Research Office (W911NF-04-1-0170).

${ }^{1}$ J. A. Rogers and Z. Bao, J. Polym. Sci., Part A: Polym. Chem. 40, 3327 (2002).

${ }^{2}$ S. R. Forrest, Nature (London) 428, 911 (2004).

${ }^{3}$ S. Wagner, S. P. Lacour, J. Jones, P. I. Hsu, J. C. Sturm, T. Li, and Z. Suo, Physica E (Amsterdam) 25, 326 (2005).

${ }^{4}$ G. M. Whitesides and B. Grzybowski, Science 295, 2418 (2002).

${ }^{5}$ K. F. Bohringer, K. Goldberg, M. Cohn, R. Howe, and A. Pisano, Proceedings of the 1998 IEEE International Conference on Robotics and Automation (ICRA), Leuven, Belgium (IEEE, Piscataway, NJ, 1998), pp. 1204-1211.

${ }^{6}$ A. Winkleman, B. D. Gates, L. S. McCarty, and G. M. Whitesides, Adv Mater. (Weinheim, Ger.) 17, 1507 (2005).

${ }^{7}$ FEMLAB 3 User's Guide (COMSOL, Los Angeles, CA, 2005), http:// www.comsol.com

${ }^{8}$ G. W. Parker, Am. J. Phys. 70, 502 (2002).

${ }^{9}$ R. Becker, Electromagnetic Fields and Interactions (Dover, New York, 1982), p. 129.

${ }^{10}$ V. J. Novick, C. R. Hummer, and P. F. Dunn, J. Appl. Phys. 65, 3242 (1989).

${ }^{11}$ G. M. Colver, J. Appl. Phys. 47, 4839 (1976)

${ }^{12}$ M. P. Pepin and H. J. Wintle, J. Appl. Phys. 83, 5870 (1998).

${ }^{13}$ C. M. Cooke, IEEE Trans. Electr. Insul. 17, 172 (1982).

${ }^{14}$ B. Techaumnat, B. Eua-arporn, and T. Takuma, J. Phys. D 37, 3337 (2004).

${ }^{15}$ M. Murayama, K. Fujibayashi, M. Matsui, and N. Murasaki, J. Electrost. 42, 143 (1997). 